\title{
Spin-excitation mechanisms in Skyrme-force time-dependent Hartree-Fock calculations
}

\author{
J. A. Maruhn, ${ }^{1}$ P.-G. Reinhard, ${ }^{2}$ P. D. Stevenson, ${ }^{3}$ and M. R. Strayer ${ }^{4}$ \\ ${ }^{1}$ Institut für Theoretische Physik, Universität Frankfurt, Max-von-Laue-Str. 1, D-60438 Frankfurt am Main, Germany \\ ${ }^{2}$ Institut für Theoretische Physik II, Universität Erlangen-Nürnberg, Staudtstrasse 7, D-91058 Erlangen, Germany \\ ${ }^{3}$ Department of Physics, University of Surrey, Guildford, Surrey GU2 7XH, United Kingdom \\ ${ }^{4}$ Physics Division, Oak Ridge National Laboratory, Oak Ridge, Tennessee 37831-6373, USA
}

(Received 26 April 2006; published 11 August 2006)

\begin{abstract}
We investigate the role of odd-odd (with respect to time inversion) couplings in the Skyrme force on collisions of light nuclei, employing a fully three-dimensional numerical treatment without any symmetry restrictions and with modern Skyrme functionals. We demonstrate the necessity of these couplings to suppress spurious spin excitations owing to the spin-orbit force in free translational motion of a nucleus but show that in a collision situation there is a strong spin excitation even in spin-saturated systems which persists in the departing fragments. The energy loss is considerably increased by the odd-odd terms.
\end{abstract}

DOI: 10.1103/PhysRevC.74.027601

PACS number(s): 24.10.-i, 25.70.Lm, 21.60.Jz

Time-dependent Hartree-Fock (TDHF) enjoyed a period of large attention in nuclear physics about 30 years ago; for reviews see, e.g., Refs. [1-3]. These early calculations delivered a great number of useful insights into the basic mechanisms of heavy-ion collisions, even with the large practical restrictions of that time concerning the model, degrees of freedom, and symmetries. However, it was soon recognized not to be as comprehensive a description as originally expected. For example, widths in the distributions of fragments and kinetic energies are systematically underestimated, a fact which had been traced back in parts to missing correlations $[4,5]$. More puzzling was that average quantities, such as fusion cross sections, did not come out all that well although they should be predictable by mean field dynamics. Already at that time there were indications that the many restrictions in the calculations spoil their predictive value and that, for example, simply the proper handling of the spin-orbit $\left(1^{*} \mathrm{~s}\right)$ force can improve the results considerably towards the experimentally observed dissipation [6,7]. Computer limitations halted these developments for a while. The subsequent dramatic advance in computational power now allows three-dimensional TDHF calculations with a full-fledged Skyrme force, without any symmetry restrictions, and for any nuclear size. Accordingly, there is a renewed interest in TDHF studies as seen from recent publications on resonance dynamics [8-10] and heavy-ion collisions [11]. The present manuscript also deals with recent 3D TDHF calculations and aims to investigate the importance of a full treatment of the $1^{*}$ s force and related dissipation mechanisms.

TDHF in a nuclear context means a time-dependent meanfield theory derived from an effective energy functional. The most widely used is the Skyrme functional which was proposed long ago as a quantitative self-consistent model for the nuclear ground state [12] and dynamics [13]. The Skyrme energydensity functional consists of free kinetic energy, Coulomb energy with exchange in the Slater approximation, and an effective-interaction part depending on density $\rho$, kinetic density $\tau, 1^{*}$ s density $\vec{J}$, current $\vec{\jmath}$, and spin density $\vec{\sigma}$, for a detailed explicit expression see, e.g., Ref. [14]. Pairing is not considered in the present case where we deal mostly with closed shell nuclei. For the purpose of later discussions, we display here the $l^{*}$ s part of the functional

$$
\begin{aligned}
& E_{\mathrm{ls}}^{\text {even }}=-\int d^{3} r\left(b_{4} \rho \nabla \cdot \vec{J}+b_{4}^{\prime} \sum_{q} \rho_{q}\left(\nabla \cdot \vec{J}_{q}\right)\right), \\
& E_{\mathrm{ls}}^{\text {(odd) }}=-\int d^{3} r\left(b_{4} \vec{\sigma} \cdot(\nabla \times \vec{J})+b_{4}^{\prime} \sum_{q} \vec{\sigma}_{q} \cdot\left(\nabla \times \vec{J}_{q}\right)\right) .
\end{aligned}
$$

The index $q \in\{p, n\}$ labels protons and neutrons. The specification of the energy-density functional fixes all that is needed for TDHF and the stationary initial states. The TDHF equations are derived by time-dependent variation with respect to the single-nucleon wavefunctions $\varphi_{\alpha}^{+}$and the corresponding stationary $\mathrm{HF}$ equation by analogous stationary variation.

The full Skyrme functional and the subsequent TDHF equations meet all symmetries of space-time, in particular invariance under Galilei transformations, a condition which must be fulfilled for a meaningful theory of heavy-ion reactions [13]. Galilean invariance imposes restrictions on the form of the odd-odd terms, i.e., those terms containing the time-odd pieces, current $\vec{j}$ and spin density $\vec{\sigma}$. This means that the kinetic term always appears in the boost-invariant combination $\rho \tau-\vec{J}^{2}$. Of particular importance here is the correct interplay between the even-even and odd-odd parts of the $l^{*}$ s term, Eqs. (1) with (2). We shall now discuss their effect on heavy-ion collisions and, in particular, show that their omission leads to inconsistencies.

The Skyrme functional allows a very precise description of nuclear ground state properties and excitations [14]. There exists, in fact, a great variety of parametrizations of the Skyrme functional in the literature which differ in quality and bias of fitted data. In order to distinguish generic effects from particularities of a certain parametrization, we considered several different Skyrme forces and show results for two: SkM$^{*}$ [15] and SLy6 [16]. Calculations with other forces did not differ significantly for the purposes of this work. 
The practical solution of the TDHF equations employs a representation of wavefunctions, potentials, and densities on a three-dimensional Cartesian coordinate-space grid. Derivatives are evaluated in Fourier-transformed space using the fast Fourier transformation (FFT) [17]. We work with a grid spacing of $1 \mathrm{fm}$. The accelerated gradient iteration is employed to find the stationary ground state solution $[17,18]$. The Coulomb field is calculated by solving the Poisson equation on a grid which is twice as large as the physical grid and with periodic boundary conditions with the method of Ref. [19]. Note that the reflection of emitted nucleons from the boundaries of the numerical box lead to an uncertainty of 2-3 MeV in the final relative-motion energy, an effect which is also seen in giant resonance calculations [20]. A Taylor series expansion up to sixth order of the unitary mean-field propagator is used for the dynamical time stepping [21]. The conservation of particle number and total energy provides a rather stringent check of numerical accuracy. In practice, we tune our numerical parameters such that we observe over all time a change in the particle number of less than 0.01 , and a drift in the total energy of less than $0.1 \mathrm{MeV}$. A time step of $\Delta t=0.2 \mathrm{fm} / c$ was found adequate in all cases considered, independent of the bombarding energy and also of whether the odd-odd $\mathrm{l}^{*} \mathrm{~s}$ couplings were included or not.

The standard test case throughout this paper is an ${ }^{16} \mathrm{O}+{ }^{16} \mathrm{O}$ collision at $E_{\text {c.m. }}=75-150 \mathrm{MeV}$. This system is one of the most frequently studied with TDHF and has also been the focus of investigations of the effect of the $1^{*}$ s force on dissipation [7]. The fragment wave functions are placed symmetrically on the grid to an initial center of mass distance of $16 \mathrm{fm}$ and then boosted to the desired relative center-of-mass (c.m.) energy. This prepares the initial state from which the TDHF propagation is calculated. We also compare results for systems including ${ }^{12} \mathrm{C},{ }^{13} \mathrm{C}$, and ${ }^{48} \mathrm{Ca}$ in order to get a first impression of the systematic variations.

The most interesting observable in heavy-ion collisions is the kinetic energy of relative motion of the two fragments. This quantity is deduced using a two-body analysis of the time-dependent density distribution. For this purpose, we calculate the principal axes of the mass quadrupole tensor, then examine the density along the axis of minimum quadrupole moment to find whether it shows the characteristics of two maxima separated by a low-density region. The point of lowest density along this line then defines a dividing plane perpendicular to this axis, and two fragments are assumed to exist on both sides of this plane. Calculating the centers of mass of each fragment yields a new straight line connecting them, which is used to repeat the process. This is iterated until the definitions of the fragment centers of mass and the dividing plane have stabilized. The principal result of this analysis are the fragment masses and charges $M_{i}, Q_{i}, i=1,2$, the separation distance $R$ of the fragments, the relative velocity $\dot{R}$ as well as the angular velocity of rotation in the scattering plane $\dot{\theta}$ calculated from the positions in two successive time steps. The energy of relative motion can then be calculated from the radial kinetic and rotational energy of the fragment minus the remaining Coulomb interaction. The Coulomb energy is approximated by the expression for two point charges, which should be good for larger distances. By comparing this

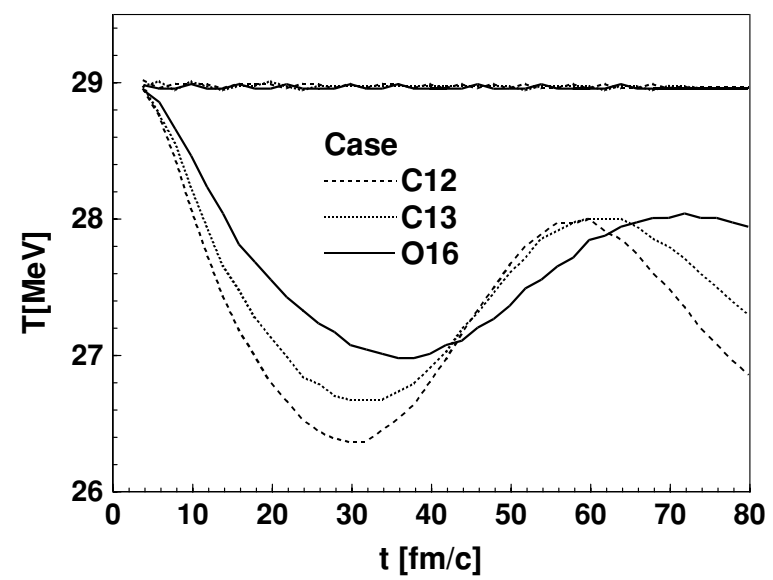

FIG. 1. The c.m. energy of a single free nucleus moving through the grid for three different test cases as indicated. The nearly constant lines correspond to the full Skyrme treatment, while the other results were calculated omitting the odd-odd $1^{*}$ s coupling.

with the full numeric calculation of the Coulomb interaction energy, we could establish that for $R \geqslant 12 \mathrm{fm}$ it is accurate within about $0.02 \mathrm{MeV}$.

As a first critical test, we consider the free translational motion of a nucleus. The results for three different nuclei shown in Fig. 1 strikingly illustrate, on the one hand, the accuracy of the code, and on the other, the need for odd-odd couplings. If the full Skyrme force is used, the kinetic energy of the c.m. just shows small oscillations by $|\Delta T|<0.2 \mathrm{MeV}$ in the period covered, while with the odd-odd couplings omitted there is an immediate deceleration followed by large oscillations. At this point we can already conclude that the omission of these terms leads to unacceptable physical behavior.

The spurious dissipation is caused by the intrinsic excitation of a spin-twist mode. For a nucleus moving with constant velocity $\vec{v}$, the coupling term contains

$$
\nabla \times \vec{J}=(\nabla \rho) \times \vec{v}=\frac{\mathrm{d} \rho}{\mathrm{d} r} \frac{\vec{r} \times \vec{v}}{r},
$$

where spherical symmetry was assumed for simplicity. This is an azimuthal vector field which thus couples to a spin field of the same character. Omitting the odd-odd coupling thus leads to a spurious excitation of a ring-like spin density, which with the full Skyrme force is suppressed by the odd-odd terms. An examination of the spin density in the cases without the odd-odd $\mathrm{l}^{*} \mathrm{~s}$ terms shows that the actual excitation of this mode accounts for about $95 \%$ of the energy loss. The rest is due to additional excitations caused by the deceleration (note that the total energy is conserved in any case).

Figure 2 shows the relative c.m. energy for a collision. The initial phase (up to $40 \mathrm{fm} / \mathrm{c}$ ) is free c.m. motion of the two nuclei, and we see again the spurious dissipation of c.m. energy as soon as the odd-odd $1^{*}$ s term is omitted. In contrast, the full Skyrme interaction preserves the relative motion energy very well until contact. The bottom part of the figure shows the energy contained in the odd-odd $l^{*} \mathrm{~s}$ term (2). The "no-odd" case shows a substantial increase in that energy which is obviously properly compensated in the full 


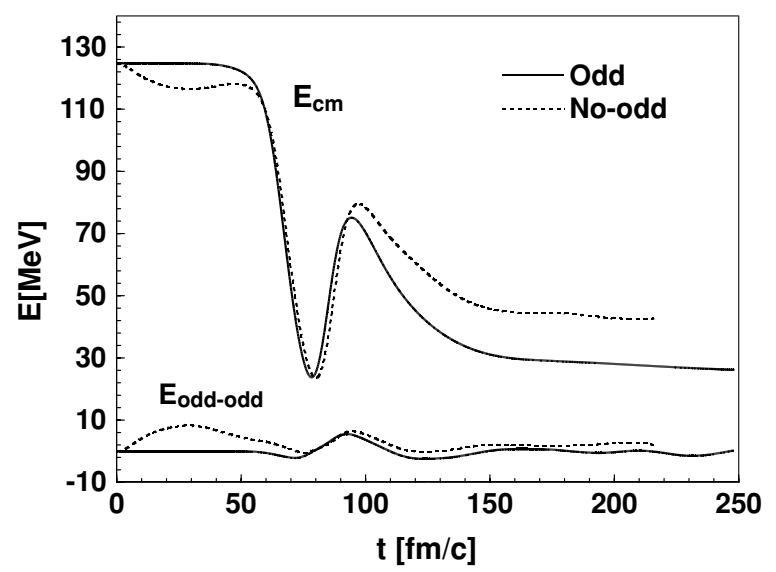

FIG. 2. Relative c.m. energy (top) and odd-odd 1*s energy (bottom) in a head-on ${ }^{16} \mathrm{O}+{ }^{16} \mathrm{O}$ collision at $125 \mathrm{MeV}$. The energies values loose their meaning in the contact regime, which is between about 50 and $120 \mathrm{fm} / c$. "odd" and "no-odd" refer to calculation with and without the odd-odd $1^{*}$ s couplings.

treatment. The energetic relations are reversed in the exit channel. The unphysical case without odd-odd $1^{*}$ s departs with more residual c.m. energy while the full interaction produces more true dissipation. This is caused again by the mechanism sketched in the spin-coupling term (3), but now is not spurious: it occurs because the $1^{*} \mathrm{~s}$ terms in the single-particle Hamiltonian and the counterbalancing odd-odd $1^{*}$ s coupling come out of synchronization due to the physical change in current pattern during the collision, so that a net spin-twist excitation remains. Figure 3 visualizes the pattern of the spin-twist excitation in a stage where the two fragments start interacting. This spin density distribution pattern was observed in the numerical results. The excitation persists after full separation. The extra amount of energy stored in this mode explains the enhanced dissipation observed in the exit stages of Fig. 2. The spin-twist mode and the unambigous detection of its deexcitation following a collision certainly deserves further detailed investigations.

We have investigated a great variety of central and noncentral collisions at various collisional energies. They all show similar effects. As one example, Fig. 4 shows the loss of c.m. energy versus impact parameter, mainly for $\mathrm{SkM}^{*}$ at

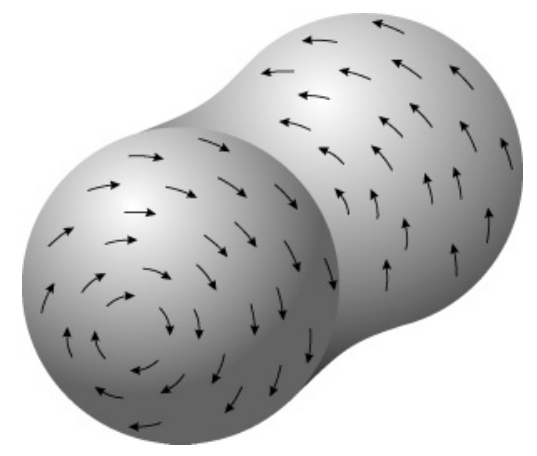

FIG. 3. Artist's view of the spin excitation generated in a central collision of two ${ }^{16} \mathrm{O}$ nuclei. Closely based on the numerical spindensity vectors produced in the calculations.

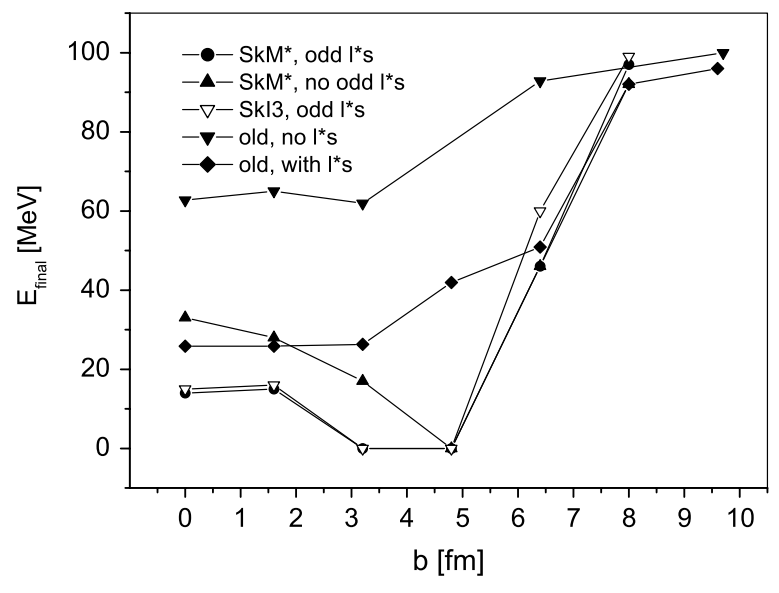

FIG. 4. The final c.m. kinetic energies in noncentral collisions of ${ }^{16} \mathrm{O}$ on ${ }^{16} \mathrm{O}$ at $100 \mathrm{MeV}$ at the impact parameters given, and with or without the inclusion of the odd-odd $l^{*} s$ terms. The Skyrme force used was $\mathrm{SkM}^{*}$. For comparison, results from Ref. [7] are shown, with and (completely) without $l^{*} s$-force.

various levels of approximation, one result from SkI3 for comparison, and results from older TDHF studies in axial approximation [6,7]. The old calculations were done with a variant of $\mathrm{SkM}^{*}$ replacing the gradient terms by finite folding. At that time, it was great success to include the even-even $1^{*}$ s term. This brought a substantial jump in dissipation and resolved the puzzle of too much transparency in the then older TDHF calculations. The present calculations without odd-odd terms still differ from the previous ones in that they are now fully triaxial. This makes no effect at low impact parameter (the minor difference is probably due to the folding approximation) but a visibly enhanced dissipation for grazing collisions which is reasonable because non-central collisions break axial symmetry and call for a triaxial treatment. The most interesting effect here is the additional dissipation caused by the step to the full Skyrme functional (compare up-triangles with full dots). It remains very similar up to an impact parameter of to about $5 \mathrm{fm}$ which is, not suprisingly, close to the nuclear radius. For larger $b$, it rapidly vanishes as we get to peripheral collisions. The spin-twist mode thus leads to excitations in the final fragments that remain relevant for a large range of impact parameters. The most striking effect is the qualitative difference that thew new calculations without symmetry restrictions and with all odd-odd terms included predict a large regime of fusion whereas all the other do not. We can estimate a fusion cross section for the system ${ }^{16} \mathrm{O}+{ }^{16} \mathrm{O}$ at collisional energy of $100 \mathrm{MeV}$ to be somewhat larger than $500 \mathrm{mb}$. This compares very well with $550 \mathrm{mb}$ deduced from the systematics of Ref. [22] while all approximate calculations fail in that respect. Fig. 4 also shows the result for full SkI3, which is very similar to $\mathrm{SkM}^{*}$. The same similarity is seen for other forces investigated. Calculations with older Skyrme forces showed a much more dramatic and systematic force dependence $[6,7,23,24]$.

The dependence of the additional dissipation (as compared to restricted calculations) on the mass of the colliding nuclei was tested by a few calculations for other collision partners. 
For ${ }^{12} \mathrm{C}$ on ${ }^{12} \mathrm{C}$ the additional energy loss was similar and possibly even larger reaching $30 \mathrm{MeV}$, and similarly for ${ }^{12} \mathrm{C}$ on ${ }^{16} \mathrm{O}$. On the other hand, for ${ }^{48} \mathrm{Ca}$ on ${ }^{48} \mathrm{Ca}$ it was almost negligible with about $1 \mathrm{MeV}$. The reason for this is not yet clear and needs to be investigated.

In this work we have investigated the energy loss in heavy-ion collisions as described by TDHF handled in full $3 \mathrm{D}$ and including consistently all terms of the given Skyrme functional. Particular attention was paid to the time reversal odd-odd spin-orbit $\left(1^{*} \mathrm{~s}\right)$ term which is often neglected in TDHF calcuations. The main findings can be concluded in brief: The odd-odd $1^{*}$ s terms establish full Galilean invariance of the functional and they are crucial to provide properly free translation of a nucleus over the grid. The odd-odd $1^{*} \mathrm{~s}$ terms add substantially to the dissipation observed in heavy-ion collisions. That effect persists up to impact parameters of order of the nuclear radius. It is large for small nuclei and seems to decrease for heavier ones. The enhanced dissipation is associated with the strong excitation of a pronounced spin-twist mode which is present even in the collision of spin-saturated nuclei and persists after separation in both fragments. The two main tasks for future research are: first, large scale investigations of dissipation under varying scattering conditions, and second, a closer inspection of that most interesting spin-twist mode, working out directions for an experimental assessment.

This work was supported by BMBF under contracts no. $06 \mathrm{~F}$ 131 and 06 ER 808 and the UK EPSRC grant GR/S96425/01. We gratefully acknowledge support by the Frankfurt Center for Scientific Computing.
[1] J. P. Svenne, Adv. Nucl. Phys. 11, 179 (1979).

[2] J. W. Negele, Rev. Mod. Phys. 54, 913 (1982).

[3] K. T. R. Davies, K. R. S. Devi, S. E. Koonin, and M. R. Strayer, in Treatise on Heavy-Ion Physics, Vol. 3 Compound System Phenomena, edited by D. A. Bromley (Plenum Press, New York, 1985), p. 3.

[4] P.-G. Reinhard, K. Goeke, and R. Y. Cusson, Nucl. Phys. A398, 141 (1983).

[5] J. B. Marston and S. E. Koonin, Phys. Rev. Lett. 54, 1139 (1985).

[6] A. S. Umar, M. R. Strayer, and P.-G. Reinhard, Phys. Rev. Lett. 56, 2793 (1986).

[7] P.-G. Reinhard, A. S. Umar, K. T. R. Davies, M. R. Strayer, and S.-J. Lee, Phys. Rev. C 37, 1026 (1988).

[8] T. Nakatsukasa and K. Yabana, Phys. Rev. C 71, 024301 (2005).

[9] J. A. Maruhn, P.-G. Reinhard, P. D. Stevenson, J. R. Stone, and M. R. Strayer, Phys. Rev. C 71, 064328 (2005).

[10] C. Simenel and P. Chomaz, Phys. Rev. C 68, 024302 (2003).

[11] A. S. Umar and V. E. Oberacker, Phys. Rev. C 73, 054607 (2006).

[12] D. Vautherin and D. M. Brink, Phys. Rev. C 5, 626 (1972).

[13] Y. M. Engel, D. M. Brink, K. Goeke, S. J. Krieger, and D. Vautherin, Nucl. Phys. A249, 215 (1975).
[14] M. Bender, P.-H. Heenen, and P.-G. Reinhard, Rev. Mod. Phys. 75, 121 (2003).

[15] J. Bartel, P. Quentin, M. Brack, C. Guet, and H.-B. Håkansson, Nucl. Phys. A386, 79 (1982).

[16] E. Chabanat, P. Bonche, P. Haensel, J. Meyer, and R. Schaeffer, Nucl. Phys. A627, 710 (1997).

[17] V. Blum, G. Lauritsch, J. A. Maruhn, and P.-G. Reinhard, J. Comput. Phys. 100, 364 (1992).

[18] P.-G. Reinhard and R. Y. Cusson, Nucl. Phys. A378, 418 (1982).

[19] J. W. Eastwood and D. R. K. Brownrigg, J. Comput. Phys. 32, 24 (1979)

[20] P.-G. Reinhard, P. D. Stevenson, D. Almehed, J. A. Maruhn, and M. R. Strayer, Phys. Rev. E 73, 036709 (2006).

[21] H. Flocard, S. E. Koonin, and M. S. Weiss, Phys. Rev. C 17, 1682 (1978).

[22] J. R. Birkelund, L. E. Tubbs, J. R. Huizenga, J. N. De, and D. Sperber, Phys. Rep. 56, 107 (1979).

[23] J. A. Maruhn, K. T. R. Davies, and M. R. Strayer, Phys. Rev. C 31, 1289 (1985).

[24] A. S. Umar, M. R. Strayer, P.-G. Reinhard, K. T. R. Davies, and S.-J. Lee, Phys. Rev. C 40, 706 (1989). 